\title{
Q-V droop control using fuzzy logic and reciprocal characteristic
}

\author{
Lu Wang ${ }^{\mathrm{a}^{*}}$, Yanting $\mathrm{Hu}^{\mathrm{a}}$, Zhe Chen ${ }^{\mathrm{b}}$ \\ ${ }^{a}$ School of Engineering and Applied Physics, Glyndwr University, Wrexham, LL11 2AW, UK \\ ${ }^{b}$ Dept. of Energy and Technology, Aalborg University, Aalborg, DK-9100, Denmark
}

\begin{abstract}
Today, the conventional power system is facing some global environmental problems, which is leading to a new trend of power grid by using "green and clear" energy sources. As the platform of smart grid technology, the microgrid associated with distributed energy resources (DERs) may provide electric power at distributed voltage level, which is not only an autonomous system, but also can be connected to the main grid. To improve the stability and controllability of the power grid, this paper presents an improved Q-V droop control strategy using fuzzy logic controller and reciprocal characteristic. Matlab/Simulink is used for analysing the performance of system. The feasibility of the improved droop control strategy has been verified and discussed. The results demonstrate the improved Q-V droop control strategy could have good effects in grid-connected and islanded mode, and during operation mode transitions.
\end{abstract}

Keywords: Microgrid, reactive power, voltage, droop control, fuzzy logic, reciprocal characteristic

\section{Introduction}

Today's world, the conventional power system is facing three main problems, including gradual depletion of fossil fuel resources, environmental pollution and poor energy efficiency. These problems have led to a new trend of power grid by using "green and clear" energy sources. Therefore, as the conglomerate of distributed generations (DGs), the microgrid technology has gained increasing attention. However, the output power of DGs is randomness, intermittent and difficult to control due to the weather condition and seasonal change, which can easily cause power quality, grid security and stability issues. Therefore, some control strategies and techniques have been studied in the last decades. Among them, droop control strategy has been a hot topic. Droop control method usually respectively regulates the frequency and voltage amplitude to balance the active power and reactive power outputted from the inverter, or simply $P-f$ and $Q-V$ droop control for short. The advantage of droop control is to make the microgrid operated in the autonomous mode and easily add/shed the additional DGs at any point to act as "plug-and-play" feature. However, the cost of traditional droop control strategy is to neglect regulation accuracy, which cannot guarantee the quality of the grid's frequency and the voltage. Therefore, some droop control strategies based on the improved droop coefficient were studied in the literatures [1]-[5].

In this paper, Q-V droop control using fuzzy logic and reciprocal characteristic are proposed. The traditional and proposed methods are discussed respectively. Finally, the performances of the proposed droop control strategies are compared and analysed based on the results produced with Matlab/Simulink.

\section{System Configuration}

The example system is a three-phase voltage-source-inverter-based radial microgrid in Fig. 1. Feeder $\mathrm{A}, \mathrm{B}$ and $\mathrm{C}$ consist of DERs interfaced by the power electronic inverters and critical loads. In the system,

\footnotetext{
* Manuscript received July 10, 2013; revised July 31, 2013.

Corresponding author. Tel.: +44-1978-293153; E-mail address: walleva1126@ hotmail.com.
} 
feeder D only includes non-critical load, DG1 and DG2 are assumed as DC power source to simulate solar photovoltaic (PV) or fuel cell, DG3 can supply the AC power to the load like wind farm or diesel. Through the point of common coupling, microgrid can be connected to or disconnected from the main power grid as per grid code. All DGs act with "plug-to-play" feature and independently control to maintain the stability and reliability of the power grid, in response to any disturbance and change maintain the stability and reliability of the power grid, in response to any disturbance and change.

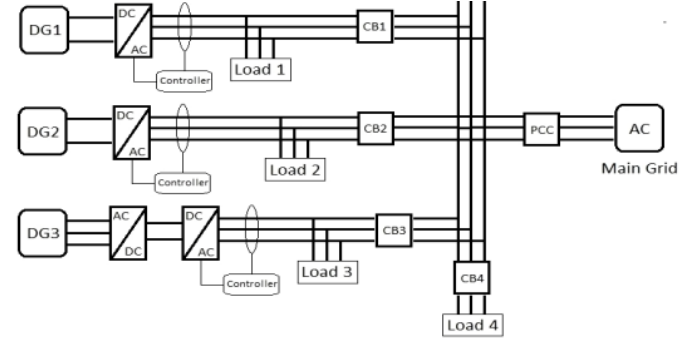

Fig. 1. The system structure.

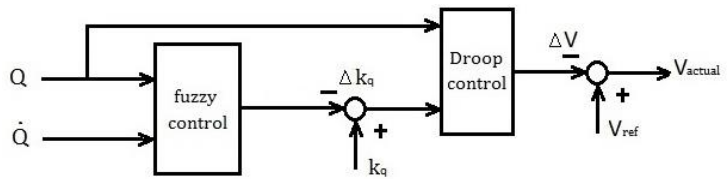

Fig. 2. Fuzzy control block.

\section{The Conventional Q-V Droop Control}

The inverter of DG controls the magnitude and phase angle of output voltage and supply the power to the grid through an inductor of reactance $X$. Normally, the phase shift $\delta$, so approximately having $\cos \delta=1$. Ignoring the resistance of transmission line, then $\mathrm{Z}=\mathrm{X}$, the reactive power equation can be represented as follows:

$$
Q=3 V_{1} V\left(V_{1}-V\right) / 2 X
$$

From (1), we can find the reactive power flow could be controlled by the voltage magnitude $V$. Therefore, the reactive power versus voltage droop characteristics can be expressed as:

$$
V_{\text {ref }}-V=-k_{q}\left(Q_{\text {ref }}-Q\right)
$$

where $V_{\text {ref }}$ indicates the reference voltage; $V$ is the actual voltage; $k_{q}$ stands for the $Q$ - $V$ droop characteristic coefficient. When the loads changed, the reactive power sharing can be done based on $k_{q}$, the system's voltage can be regulated, then reach to a new steady-state level. Conventionally, the simple droop coefficient is set to be a constant [6]-[9]. As the reactive output power changing, the voltage magnitude of the grid can be changed correspondingly and have a quick response to keep stable. However, the output power of DG may not be constant affected by the weather condition, seasonal change and regional conditions. If $k_{q}$ is set to a big value, a small reactive power change will lead to a greater voltage deviation, which may exceed the allowed fluctuation range referred to IEEE 1547 Standard. Therefore, in order to cope with the power variation, the variable droop coefficient would have more advantages.

\section{The Proposed $Q-V$ Droop Control}

\subsection{Fuzzy logic}

The fuzzy logic control design is composed of three sections: fuzzification, inference engine and defuzzification. In this case, the error of reactive output power from DGs (inputs 1) and the change rate of error (inputs 2) are used as inputs to the fuzzy reasoning. The fuzzy output is the adjustment of $Q$ - $V$ droop coefficient in Fig. 2. Membership function of the input 1 and output are assigned to seven fuzzy subsets: negative big (NB), negative medium (NM), negative small (NS), zero (ZO), positive small (PS), positive medium (PM), and positive big (PB). Membership function of input 2 is designed to five fuzzy subsets: NB, NS, ZO, PS and PB. The shape of membership function adapts the triangular. The value of the two inputs and output are normalized by the scaling factor. The input and output scaling has designed between 0 and 1. 
Table 1. Rule of fuzzy reasoning

\begin{tabular}{|c|c|c|c|c|c|c|c|c|}
\hline \multirow{2}{*}{\multicolumn{2}{|c|}{ Output }} & \multicolumn{7}{|c|}{ Input 1} \\
\hline & & PB & PM & PS & $\mathrm{ZO}$ & NS & NM & NB \\
\hline \multirow{5}{*}{ Input2 } & PB & PB & PM & PM & PS & $\mathrm{ZO}$ & NS & $\mathrm{NB}$ \\
\hline & PS & PB & PM & PS & $\mathrm{ZO}$ & $\mathrm{ZO}$ & NS & NB \\
\hline & $\mathrm{ZO}$ & PB & PM & PS & $\mathrm{ZO}$ & NS & NM & NB \\
\hline & NS & PB & PS & $\mathrm{ZO}$ & $\mathrm{ZO}$ & NS & NM & NB \\
\hline & NB & PB & PS & $\mathrm{ZO}$ & NS & NM & NM & NB \\
\hline
\end{tabular}

The fuzzy reasoning is described by a set of "if-then" rule. Based on the levels of the two input signals, the adjustment of droop coefficient will be obtained. The rules of fuzzy reasoning are described in Table 1. The adjustment value of droop coefficient can be calculated with defuzzificaion using the centroid method. This method can fully consider the information of fuzzy reasoning, which is described by the area surrounded by the membership function curve and the horizontal axis. When the output variables of the membership function is a set of discrete points, then:

$$
\Delta k=\frac{\sum_{i=1}^{m} x_{i} \mu_{N}\left(x_{i}\right)}{\sum_{i=1}^{m} \mu_{N}\left(x_{i}\right)}
$$

where $\Delta k$ is the output variable; $m$ is the number of rule; $\mu_{N}$ is the grade of membership; $x_{i}$ is defined as a discrete universal set. By this way, the output of $\Delta k$ can be calculated to a precise value with defuzzificaion. Therefore, the actual droop coefficient $k_{q}$ is equal to:

$$
k_{q_{-} \text {actual }}=k_{q}-\Delta k_{q}
$$

\subsection{Reciprocal characteristic}

The representation of the reciprocal curve is selected as (5), in which $m$ and $A$ should be positive constants; otherwise the function becomes divergence with slope being positive as shown in Fig. 3 .

$$
y=A /\left(x^{m}+1\right),(m>0 \text { and } A>0)
$$

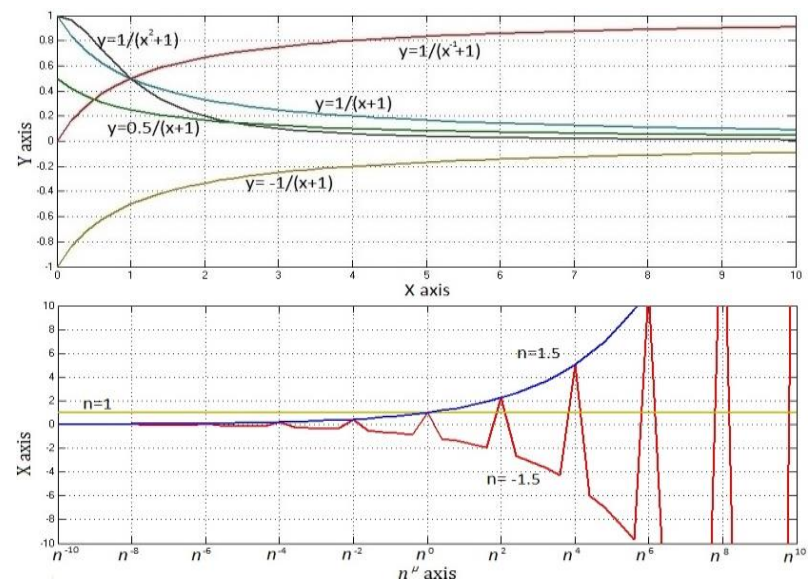

Fig. 3. The curves $y=A /\left(x^{m}+1\right)$ and $x=n^{\mu}$.

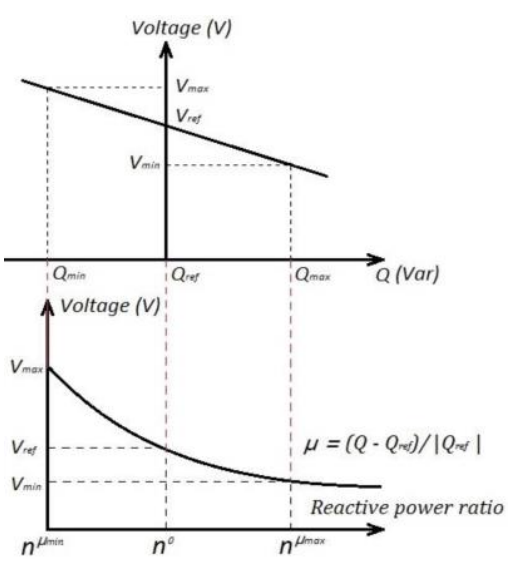

Fig. 4. The relationship between $Q$ and $\mu$.

It can be noticed there are five features on (5): 1) the slope of curve keeps negative at any point; 2) with the increment of $x$, the function y decreases and tends to $0 ; 3$ ) the range of function $y$ is determined by the numerator $A ; 4)$ when the value of $A$ determined, the function y always meets at $x=1$; 5) the variable $\mathrm{n}$ is bigger, $\mathrm{y}$ reduces faster around $x=1$. When in the grid-connected mode, the system voltage is supported by the main power grid. All DGs work at the reference value. Based on the feature 4), the reference value will be set up at $x=1$ point. Similar as the principle of Bode diagram, transfer the value of 
horizontal axis into the form of power function. The power is defined as $\mu$ and has a relationship with the reactive power, see Fig. 4:

$$
x=n^{\mu},(n>1) ; \mu=\left(Q-Q_{\text {ref }}\right) /\left|Q_{\text {ref }}\right|
$$

where $n$ is constant, greater than 1 . Furthermore, the deviation of voltage must satisfy the demand of grid code to keep the whole power system reliable and stable in grid-connected mode, islanded mode and during the transition. As per grid code, the deviation is assumed as $\pm 3 \mathrm{~V}$, so the value of y fluctuates within the range of 6 . Therefore, the expression (5) can be rewritten as:

$$
y=6 /\left(x^{m}+1\right)
$$

in which the medial value of $\mathrm{y}$ is equal to 3 . To achieve the value of $\mathrm{y}$ to 311 at $x=1, \mathrm{y}$ is offset by (311-3) units. Therefore, a new reciprocal curve expression is as follows:

$$
y-(311-3)=6 /\left(x^{m}+1\right),(m>0)
$$

Synthesizing the equation (6), (7) and (8), the reactive output power versus frequency droop coefficient based on reciprocal characteristic is described as:

$$
Q-(311-A / 2)=A /\left(x^{m}+1\right),(m>0 \text { and } A>0) ; x=n^{\mu},(n>1) ; \mu=\left(Q-Q_{\text {ref }}\right) /\left|Q_{\text {ref }}\right|
$$

where $Q$ stands for the actual voltage; $A$ is the range of deviation. Fig. 5 and Fig. 6 describe different $Q-V$ droop coefficients using reciprocal characteristic. In pictures, the fluctuation of voltage magnitude is from $308 \mathrm{~V}$ to $314 \mathrm{~V}$. With the increment of $m$ and $n$, the reciprocal curve has a faster trend to close to the boundary. The slope of crossing point becomes greater as well. As the same reactive power changing, the variables $m$ and $n$ are set bigger, the deviation of system voltage magnitude will be larger. The dynamic response of system also becomes quicker. Therefore, when the reactive power output is smaller, we can choose a big $m$ and $n$ to improve the system's operation. If the output power is large, the small values of $m$ and $n$ can be chosen to reduce the deviation of system's frequency.

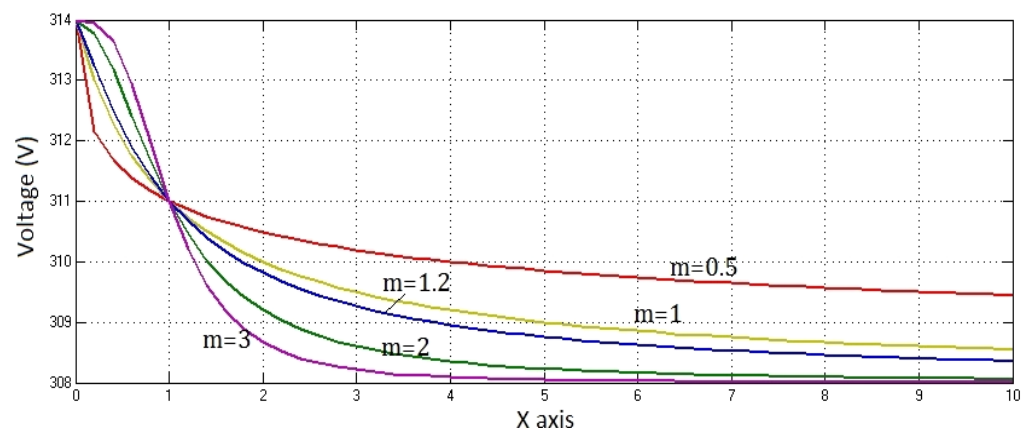

Fig. 5. Droop coefficients with $m$ increasing.

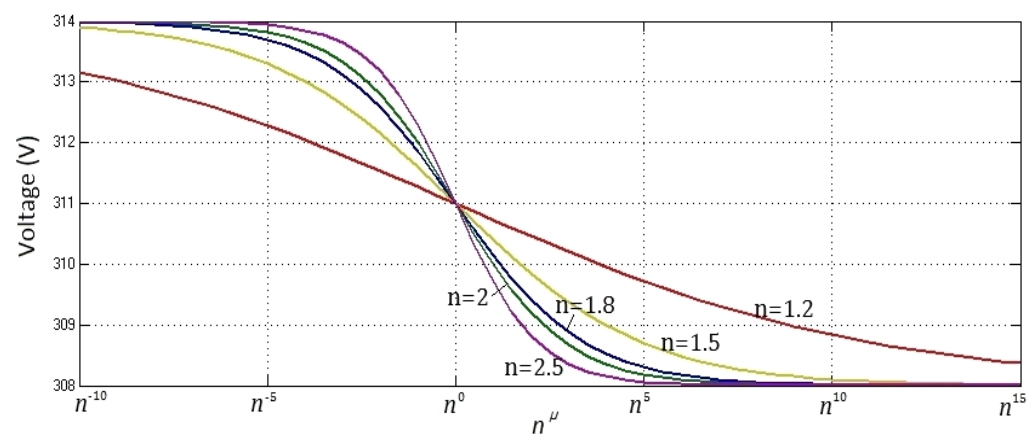

Fig. 6. Droop characteristics with $n$ in turn. 


\section{Simulation and Analysis}

In further discussions of droop control, the peer-to-peer method is adopted. All DGs may take part in droop control based on pre-set control mode. Considering different changes of system's structure, the feasibility of the droop control strategies proposed in previous section will be tested.

\subsection{Case 1}

The target of Case 1 was to verify the superiority of fuzzy logic droop control. In this model, DG3 was isolated. DG1 respectively applied with fuzzy logic and traditional droop control. To make the same initial condition, the traditional droop coefficient of DG1 was set to $2 \times 10^{-4}$. DG2 was operated by the traditional droop characteristic, $k_{q}=1.5 \times 10^{-4}$. Based on the same reactive output power changing, we could find the voltage magnitude controlled by fuzzy logic was more stable than that of traditional one during the whole process. The deviation of voltage was only $\pm 2.5 \mathrm{~V}$. At $0.5 \mathrm{~s}$, the peak value dropped from $316.5 \mathrm{~V}$ to $312.5 \mathrm{~V}$, see Fig. 7.

\subsection{Case 2}

For testing the feasibility of the proposed control strategy, both DG1 and DG2 were applied the fuzzy logic droop control with different regulations. The system structure was same as Case 1. Based on the same initial droop coefficients, the range of regulation of DG1 was $0 \sim 1.5 \times 10^{-4}$; that of DG2 was $0 \sim 1.2 \times 10^{-4}$. Table 2 outlined the change of reactive power among DG1, DG2 and non-sensitive load. When the microgrid worked in islanded mode, the reactive power of the non-sensitive load was 2190Var. At $1 \mathrm{~s}$ and $2 \mathrm{~s}$, the load was removed and then added. The inverters of DGs had a quick response to reach a new balance point and share the change of reactive power by different fuzzy logic droop characteristics. The voltage magnitude still maintained within the allowed fluctuation $\pm 3 \mathrm{~V}$, depicted in Fig. 8. The whole system kept stable and reliable to achieve the safe connection.

Table 2. Rule of fuzzy reasoning

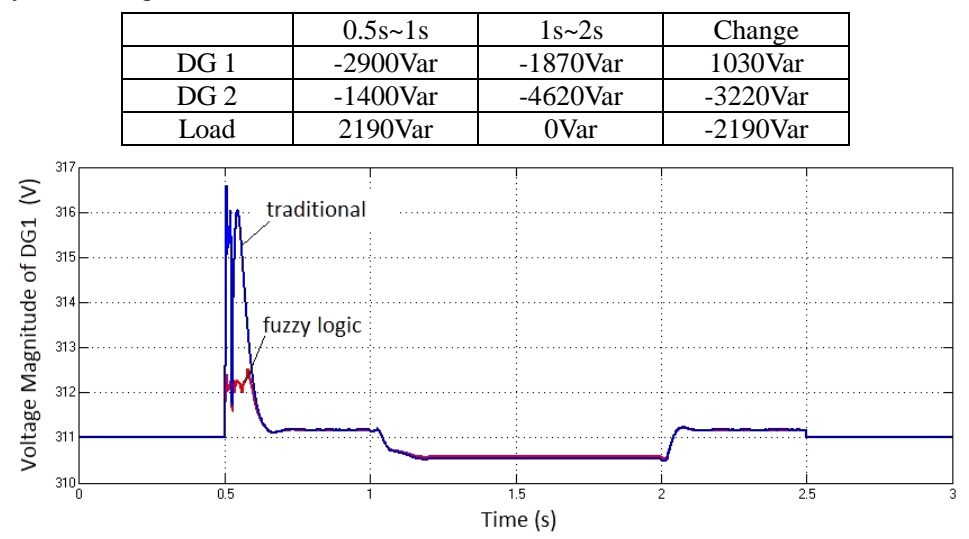

Fig. 7. DG1's voltage using different droop control.

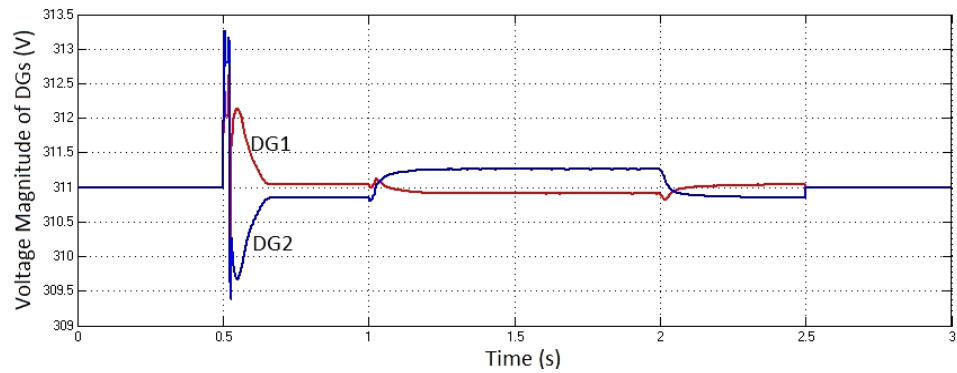

Fig. 8. Voltage magnitude of DGs in Case 2. 


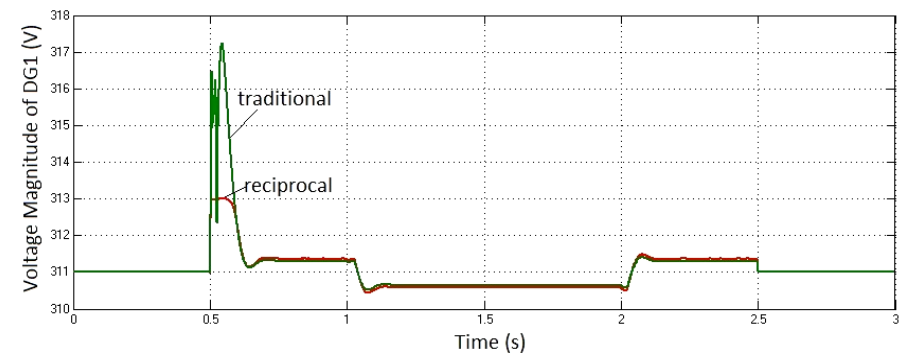

Fig. 9. Voltage magnitude of DG1 in Case 3.
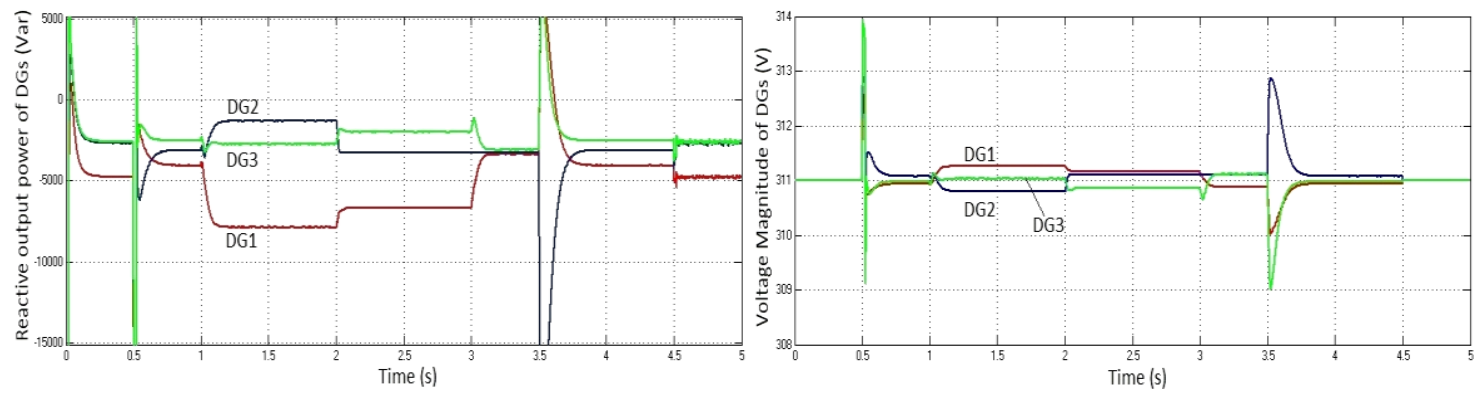

Fig. 10. Reactive output powers and voltage magnitudes of DGs in Case 4.

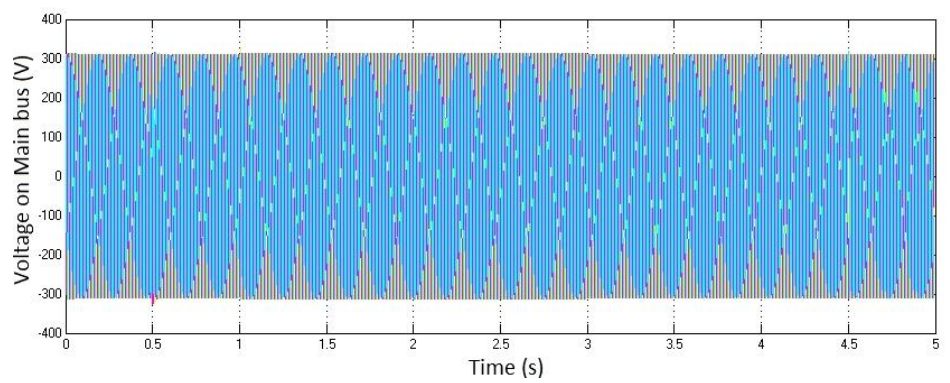

Fig. 11. The system voltage on the main bus in Case 4 .

\subsection{Case 3}

In this case, the droop control using reciprocal characteristic was discussed. Similar as Case 1, the DG1 respectively was operated by reciprocal characteristic and traditional droop control. The advantage of reciprocal characteristic is the deviation of voltage magnitude can be predetermined by the exporter. Based on the aforementioned definition, the parameters were set as $A=4, m=1$ and $n=1.5$. The simulation result was outlined in Fig. 9. In comparison of conventional method, when the range of deviation is fixed, however the reactive output power of DG1 changed, the system voltage would be floating from $310.5 \mathrm{~V}$ to $313 \mathrm{~V}$, which was not exceeded the pre-set limitation, see Fig. 9.

\subsection{Case 4}

To test the feasibility and reliability of droop control using reciprocal characteristic, three DGs need to take participate in Case 4. The pre-set deviations of DG1 and 2 were $\pm 2 \mathrm{~V}$, smaller than that of DG $2 \pm 3 \mathrm{~V}$. During the whole operating period, the non-critical load and DG2 were shed respectively and switched on again. In Fig. 10, the system transferred to the islanded operation mode at $0.5 \mathrm{~s}$. The overshoot of DG2's reactive power changing was same as than that of DG3, but the fluctuation of voltage was not exceeded the desired limitation. At 3.5s, the load and DG2 added. Based on the different predetermined parameters, the regulation of DG3's voltage was greater than that of DG1. Fig. 11 demonstrated the system voltage on the main bus. The result verified that the improved droop control strategy had a significant ability to maintain the system stability in grid-connected and islanded mode, and during operation mode transitions. 


\subsection{Case 5}

In Case 5, the range of voltage deviation was set to $\pm 3 \mathrm{~V}$. DG1 was controlled by different reciprocal droop characteristic, whose value of $m$ and $n$ increased in turn. The system structure and operating set were same as Case 3 . When the system reached a new voltage point to balance the power sharing, the fluctuation of DG's voltage magnitude becomes bigger due to the large $m$ and $n$. Therefore, based on the same conditions of DGs, the small $m$ and $n$ could improve the stability and reliability of the power grid, this effect could be seen in Fig. 12.
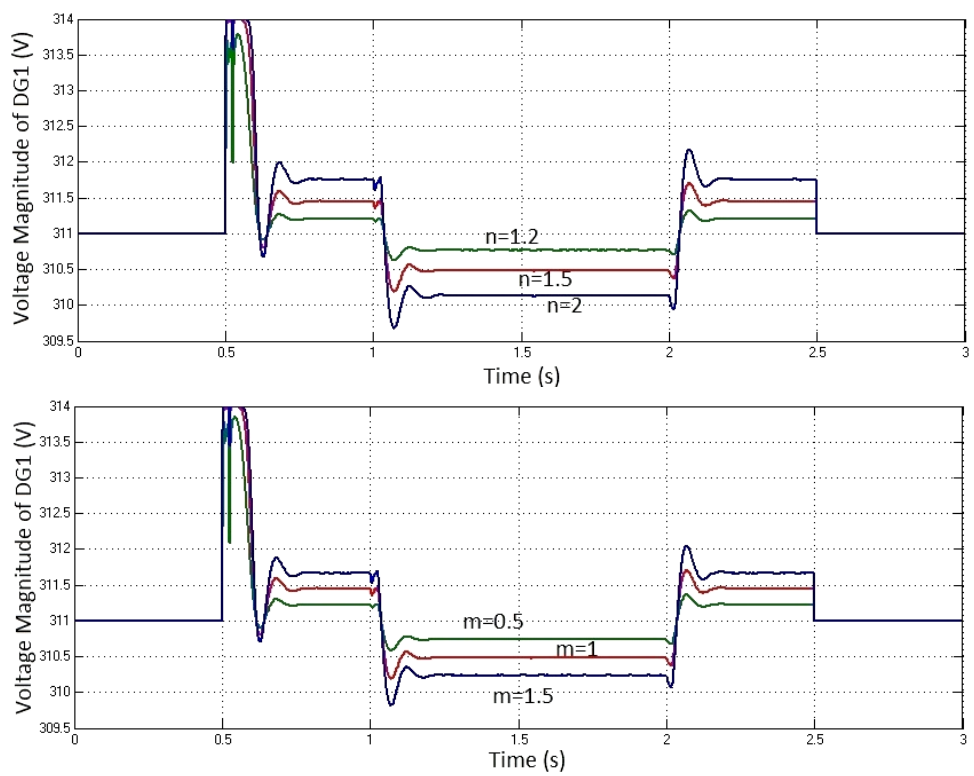

Fig. 12. Voltage magnitude of DG1 with variable $m$ and $n$.

\section{Conclusion}

In this paper, the three-phase radial microgrid model using fuzzy logic and reciprocal droop control has been discussed. For traditional droop, the small $Q-V$ droop coefficient would lead to the small system frequency deviation and a quick system dynamic response. The system could maintain stable and reliable to share the power during the mode transition and load changing. In comparison of fixed droop coefficient, the fuzzy logic droop control could achieve the droop coefficient has a dynamic change. Due to the changes of DG's reactive output power, the fuzzy controller can calculate the suitable adjustment to modify the droop coefficient. By this way, the deviation of system voltage could be guaranteed. The stability of grid would have a significant improvement. For the reciprocal droop control, the simulation result verified the range of system's voltage floating deviation could be pre-set by the operator. With the increment of variable $\mathrm{m}$ and $\mathrm{n}$, the regulation of system voltage is greater with the same change of reactive output power from DGs. The dynamic response of system also became faster. Finally, the feasibility and effectiveness of different control methods and the synthesized operation models were tested. The results demonstrated that the improved droop control strategy performs well in grid-connected and islanded mode, and during operation mode transitions.

\section{References}

[1] Oshiro M, Uchida K, Senjyu T, Yona A. Voltage control in distribution systems considered reactive power output sharing in smart grid. In: Proc. of 2010 International Conference on Electrical Machines and Systems (ICEMS), 2010:458-463.

[2] Li P, Wang W, Yang X, Wang S, Cui H, Gao C. A droop control method of microsources based on divided self-adjusting slope coefficient. In: Proc. of 2010 International Conference on Power System Technology (POWERCON), 2010:1-6. 
[3] Sun G, Zha X. Research on parallel operation of distributed inverter based on variable droop coefficient. In: Proc. of 2011 International Conference on Electric Information and Control Engineering (ICEICE), 2010:4411-4414.

[4] Zhu J, Li P, Wang Y, Chen C. Microgrid power control method based on improved droop characteristics. Power System and Clean Energy, 2012; 28(6):29-33 (in Chinese).

[5] Chaudhuri NR, Chaudhuri B. Adaptive droop control for effective power sharing in multi-terminal DC (MTDC) grids. IEEE Transactions on Power Systems, 2013; 28(1):21-29.

[6] Barklund E, Pogaku N, Prodanovic M, Hernandez-Aramburo C, Green TC. Energy management in autonomous microgrid using stability-constrained droop control of inverters. IEEE Transactions on Power Electronics, 2008; 23(5):2346-2352.

[7] Su L, Li G, Jin Z. Modeling, control and testing of a voltage-source-inverter-based microgrid. In: Proc. of the 4th International Conference on Electric Utility Deregulation and Restructuring and Power Technologies (DRPT), 2011:724-729.

[8] Zhi N, Zhang H, Liu J. Overview of microgrid management and control. In: Proc. of International Conference on Electrical and Control Engineering (ICECE), 2011:4598 - 4601.

[9] Alotibe MS, Chandrasena R, Rajakaruna S. Simplified voltage and frequency controller based on droop control for the dynamic analysis of a Microgrid. In: Proc. of the 22nd Australasian Universities Power Engineering Conference (AUPEC), 2012:1-8.

[10] Chen Z, Hu Y. Control of power electronic converters for distributed generation units. In: Proc. of the 31st Annual Conference of the IEEE Industrial Electronics Society, 2005:1317-1322.

[11] Hu Y, Chen Z, McKenzie H. Voltage source converters in distributed generation systems. Presented at: The Third International Conference on Electric Utility Deregulation and Restructuring and Power Technologies, 2008.

[12] Chen Z, Hu Y, McKenzie H. Effects of line parameters on performance of voltage source converters in distributed generation systems. Presented at: The 43rd International Universities Power Engineering Conference UPEC 2008. 